\title{
Upregulation of LncRNAs THRIL and Malat1 in Peripheral Blood of Ischemic Stroke Patients with Large Artery Atherosclerotic and Small Vessel Disease
}

\section{Mahnaz Bayat}

Shiraz University of Medical Sciences

Najmeh Karimi

Shiraz University of Medical Sciences

Moosa Rahimi

Shiraz University of Medical Sciences

Reza Tabrizi

Shiraz University of Medical Sciences

Tahereh Asadabadi

Shiraz University of Medical Sciences

Etrat Hooshmandi

Shiraz University of Medical Sciences

Mohammad Saied Salehi

Shiraz University of Medical Sciences

Seyedeh Shaghayegh Zafarmand

Shiraz University of Medical Sciences

\section{Maryam Owjfard}

Shiraz University of Medical Sciences

\section{Mahintaj Dara}

Shiraz University of Medical Sciences

\section{Carlos Garcia Esperson}

Shiraz University of Medical Sciences

Neil Spratt

Shiraz University of Medical Sciences

\section{Christopher Levi}

Shiraz University of Medical Sciences

Afshin Borhani haghighi ( $\nabla$ neuro.ab@gmail.com )

Shiraz University of Medical Sciences https://orcid.org/0000-0002-4131-7990 


\section{Research Article}

Keywords: Atherosclerosis, Long non-coding RNA, Malat1, THRIL, Ischemic stroke

Posted Date: November 30th, 2021

DOI: https://doi.org/10.21203/rs.3.rs-1065192/v1

License: (c) (i) This work is licensed under a Creative Commons Attribution 4.0 International License. Read Full License 


\section{Abstract}

\section{Background}

Long non-coding RNA (IncRNA) has the main role in gene regulation and it might serve as a potential biomarker in clinical practice. Malat1 and THRIL LncRNAs have been demonstrated to play key roles in inflammation and atherosclerosis. It was hypothesized that the Malat1 and THRIL expression increase in patients with atherosclerotic ischemic stroke (IS) with significant diagnostic value for discriminating IS from controls.

\section{Methods}

We evaluated Malat1 and THRIL expression on days 1,3, and 5 after stroke in 59 IS cases with smallvessel disease (SVD) and large artery atherosclerosis (LAA), and 63 controls. A real-time polymerase chain reaction was used for the evaluation of IncRNA expression.

\section{Results}

In patients with SVD or LAA, Malat1 and THRIL expression significantly were higher than the controls and mix model analysis showed significantly higher expression of IncRNAs on days 5 relative to days 1 and 3 after stroke while the positive correlation was also detected between Malat1 expression and time after stroke $(r=0.27, p=0.03)$. After logistic regression analysis, elevated Malat1 and THRIL showed a significant positive association with the risk of SVD and LAA. We found Malat1 could be used as a diagnostic marker with an area under the curve of $0.78(p<0.001)$.

\section{Conclusion}

This was the first study that demonstrated the significant upregulation of THRIL from 24 hours after IS until 5 days. This upregulation may serve as a biomarker for the diagnosis of IS. To reach a reliable conclusion we need a larger sample size.

\section{Introduction}

Ischemic stroke (IS) is an acute neurological disorder with poor functional outcomes it also has the second rank among leading causes of death worldwide. The long-term severe disability after ischemic stroke for patients and their families is highly problematic [1]. At the moment, we don't have a rapid, costeffective, specific, and sensitive biomarker test to confirm an imaging and clinical diagnosis of ischemic stroke. Many studies are going to develop a reliable test for IS in clinical practice [2].

Long non-coding RNAs (IncRNAs) are RNAs with more than 200 nucleotides that are not translated into functional proteins [3]. LncRNAs can play a relevant role in the modulation of biological functions, therapeutic targets and serve as a potential biomarker $[4,5]$. IncRNAs have been also shown regulatory roles in different brain cell types involved in IS pathophysiology [6]. In recent years, one of the most 
widely studied nuclear-restricted IncRNAs is Metastasis Associated Lung Adenocarcinoma Transcript 1 (Malat1). The Malat1 gene is encoded on human chromosome 11q13.1 [7]. This is an RNA gene and its transcript has been confirmed as a non-coding RNA with a good diagnostic value in different types of human cancers [8]. Malat1 was first identified in tumor tissue from patients with non-small cell lung cancer [9], and the relative upregulation of Malat1 has been associated with metastasis in lung, liver, and breast cancers [9, 10], but also diabetes [11], angiogenesis [12] or animal models of cerebral ischemia [13]. Several studies demonstrated IncRNA Malat1 could promote inflammation [14-17], and coronary atherosclerosis disease (CAD) $[18,19]$. Malat1 accelerates the accumulation of cholesterol-filled foam cells in blood vessels and enhances lipid uptake in macrophages by CD36 expression and subsequently promotes the atherosclerosis process [20]. Recent studies have identified the overexpression of Malat1 in patients with CAD while has shown its predictive value for in-stent restenosis, positive correlation with inflammatory factors, and regulation of coronary slow flow endothelial dysfunction $[18,19,21]$.

Another IncRNA that has been demonstrated to play key roles in inflammation and atherosclerosis is THRIL (Tumor necrosis factor (TNF) and heterogeneous nuclear ribonucleoprotein L (hnRNPL)- related immunoregulatory). THRIL can modulate inflammation [22, 23], and its upregulation has been found in sepsis [24], coronary heart disease [25], osteosarcoma [22], rheumatoid arthritis [26], and brain ischemia model [27]. THRIL has proved to increase the development of coronary atherosclerotic heart disease in endothelial progenitor cells [28].

Several studies have demonstrated the significant relations of Malat1 and THRIL in the pathogenesis of inflammation, atherosclerosis, and endothelial cell dysfunction [28-35] and a significant increase of Malat1 and THRIL have been also reported in the peripheral blood of atherosclerotic patients with CAD $[18,21,25,36]$. Nevertheless, their expression in IS, especially in atherosclerotic stroke is still uncertain, with only one study analyzing the blood levels of Malat1 in IS patients (all etiologies) within 24 hours from onset [37]. According to previous studies, the mortality rate of IS patients [38,39] and the rate of atherosclerosis and atherosclerotic diseases among Iranian people are higher in comparison to western countries [40]. Therefore, in this study, we evaluated the expression levels of two key IncRNAs associated with atherosclerosis and inflammation (Malat1 and THRIL) in peripheral blood of confirmed large artery atherosclerotic (LAA) and small vessel diseases (SVD) IS patients, to determine their expression levels at different time points on days $(1,3$, and 5$)$ after the event and to correlate these levels with the clinical and laboratory data of the patients as well as their potential as diagnostic biomarker.

\section{Materials And Methods 2.1 Participants}

This study was conducted from August 2019 to 2020 at Namazi Hospital in Shiraz including IS patients and keeping a control population. The inclusion criteria for stroke were: patients older than 18 years, with confirmed small-vessel disease (SVD) or large artery atherosclerosis (LAA) according to TOAST 
classification [41], admission within $24 \mathrm{~h}$ after symptom onset, and able to perform all the samples of the study. Ischemic stroke was defined as an acute neurologic deficit lasting more than 24 hours (or lasting < 24 hours with imaging evidence of ischemic infarct) [34]. All patients underwent brain non-contrast computed tomography (CT), or diffusion-weighted magnetic resonance imaging. Patients who had suffered from a transient ischemic attack without radiologic confirmation, severe inflammation, and treated with an immunosuppressant. The control subjects were from Shiraz's population while their sex and age were matched with cases all of them had at least three vascular risk factors without a history of active malignancy. The ages of the examined subjects ranged from 32-90 years. In this study, hypertension was diagnosed according to these criteria: blood pressure $\geq 140 / 90 \mathrm{mmHg}$ on two consecutive occasions at least $24 \mathrm{~h}$ apart or receiving antihypertensive therapy [42]. Diabetes mellitus was diagnosed based on the following criteria: two fasting glucose $>126 \mathrm{mg} / \mathrm{dl}(7.0 \mathrm{mmol} / \mathrm{L})$ and $2-\mathrm{h}$ post-load glucose $>200 \mathrm{mg} / \mathrm{dl}(11.1 \mathrm{mmol} / \mathrm{L})$ or receiving treatment with hypoglycemic drugs [43]. The National Institutes of Health Stroke Scale (NIHSS) score was evaluated on admission, with higher scores indicating greater severity [44]. The outcomes were obtained three months after admission according to the modified Rankin scale (mRS) blinded to the level of IncRNA [45]. Ethics approval for this study was obtained by the local ethics committee of Shiraz University of Medical Sciences (IR.SUMS.REC.1398.17988). Written informed consent was provided from all subjects (or their proxy respondents). Peripheral venous blood samples were collected from patients in 3 times after onset of stroke [day $1(n=59)$, day $3(n=43)$, and day $5(n=25)$ after stroke].

\subsection{RNA extraction and real-time-polymerase chain reaction}

The first total RNA was extracted from blood specimens by using TRIzol Reagent (GeneAll, Seoul, South Korea) according to the acid guanidium thiocyanate-phenol-chloroform method. Then CDNA was produced using the OneStep RT-PCR Kit (Add bio, Seoul, South Korea) from RNA samples with the A260/A230 and A260/A280 ratios greater than 1.8 according to the manufacturer's instructions. Relative expressions (RE) of $A N R I L$ and MALAT1 were measured in all specimens using the RealQ Plus 2x Master Mix Green High ROX ${ }^{\mathrm{TM}}$ (Add bio, Seoul, South Korea) and reactions were conducted in StepOnePlus ${ }^{\mathrm{TM}}$ RealTime PCR equipment (Applied Biosystems, Foster city, CA, USA) in duplicate with the thermal-cycling settings of $10 \mathrm{~min}$ at $95^{\circ} \mathrm{C}$ ( 1 repeat) accompanied by 40 cycles for $15 \mathrm{~s}$ at $95^{\circ} \mathrm{C}, 25 \mathrm{~s}$ at $60^{\circ} \mathrm{C}$ and $25 \mathrm{~s}$ at $72^{\circ} \mathrm{C}$ were used. Every complete amplification phase was accompanied by a melting phase for $15 \mathrm{~s}$ at 95 $\mathrm{C}, 25 \mathrm{~s}$ at $60^{\circ} \mathrm{C}$, and $15 \mathrm{~s}$ at $72^{\circ} \mathrm{C}$. GAPDH gene was used as a normalizer. The results were calculated with the $2^{-\triangle C t}$ method [46]. The primer sequences are used as follows:

\section{Malat1:}

- Forward: 5'-TCAGTGTTGGGGCAATCTT-3'

- Reverse: 5'- CGTTCTTCCGCTCAAATCC-3' 


\section{THRIL:}

- Forward:5'-TGTGATCCATACTCCTCGG-3'

- Reverse: 5'-AGGCAAGGGAGTTTCAGAA-3'

\section{GAPDH:}

- Forward: 5'-GCATCTTCTTTTGCGTCG-3'

- Reverse: 5'-TGTAAACCATGTAGTTGAGGT-3' [46].

\subsection{Statistics}

A comparison between categorical data was performed using a chi-square test. The differences between numeric variables were evaluated using an independent two-sample t-test. We used logistic regression analyses between case and controls to evaluate the association of IncRNAs expression levels and clinical parameters with the risk of IS. The significant time interaction for Malat1 and THRIL expression was estimated by mixed model analysis after adjusting the group. The IncRNAs expression level was shown as mean \pm SE. The relationship between IncRNAs levels with clinical parameters was analyzed using linear regression. We also analyzed the correlation between NIHSS and IncRNA expression by Spearman correlation. The ROC curve analysis was used to estimate the diagnostic and prognostic values, and the findings were shown as the area under the curve (AUC). The analyses were done using the SPSS software (version 19.0) and GraphPad Prism 5.0. The p-value of $<0.05$ was regarded as statistically significant.

\section{Results}

\subsection{Demographic and clinical characteristics of IS patients and controls}

A total of 59 IS patients and 63 controls were included in the study. Vascular risk factors such as diabetes and hypertension were more prevalent in cases compared to controls $(p<0.05)$ (Table 1$)$. In laboratory results, there were no significant differences in the levels of total cholesterol, triglyceride, and LDL between IS cases and controls; however, high-density lipoprotein level was significantly lower in IS cases compared to controls

\subsection{The levels of Malat1 and THRIL IncRNAs in peripheral blood of IS patients in different times points}


In the present study, we evaluated Malat 1 and THRIL expression on days 1,3, and 5 after stroke, in 59, 43, and 25 IS cases respectively, and 63 controls. Blood sampling was repeated from 25 cases on days 1,3, and 5 after stroke. We had both random and fixed cases at different times thus we used mixed model analysis. The significant time interaction was detected for Malat1 and THRIL expression after adjusting the group. The level of THRIL on days 1 and 3 significantly was lower than the expression on day 5 respectively and the Malat 1 level on day 1 significantly was lower than the expression on day 5 (Table 2). By logistic regression analysis, increasing Malat1 and THRIL levels showed a significant association with IS. After adjusting for relevant clinical and laboratory variables (age, sex, BMI, hypertension, hyperlipidemia, diabetes mellitus, smoking), elevated Malat1 and THRIL levels remained significant ( $p=$ $0.001 ; \mathrm{OR}=1.52 ; 95 \% \mathrm{Cl}, 1.18-1.96)$ and $(p=0.03 ; \mathrm{OR}=1.26 ; 95 \% \mathrm{Cl}, 1.01-1.57)$.

Malat1 expression in IS patients significantly was higher than the controls at different time points on day $1(4.1 \pm 0.65 v s 0.63 \pm 0.16)$, day $3(6.7 \pm 1.4 v s 0.60 \pm 0.20)$ and day $5(6.5 \pm 1.3 v s 1.1 \pm 0.36)$ respectively (All $p<0.001$ ) (Fig. 1A). On the first day after stroke, the Malat1 IncRNA level was higher than the control and this upregulation remained at this high level on days 3 and 5 after stroke onset.

THRIL expression in IS patients significantly was higher than the controls on day 1 (3.16 \pm 0.79 vs $0.7 \pm$ $0.14)$, day $3(3.9 \pm 1.1$ vs $0.71 \pm 0.17)$ and day $5(9.3 \pm 2.7 v s 2.6 \pm 1.8)$ respectively (All $p<0.01)$. (Fig. 1B). There were no significant differences in the expression of two IncRNAs between LAA and SVD patients (Fig. 1C and D).

\subsection{Association of Malat1 and THRIL expression with Clinical parameters}

We used linear regression analysis to detect the association between the IncRNAs levels with clinical parameters and types of stroke in the 59 patients (Table 3). There were no significant correlations between Malat1 and THRIL expression with clinical parameters and type of stroke (LAA and SVD) in IS patients. We found a non-significant negative correlation between Malat1 expression and NIHSS (Beta = $-0.333, p=0.12$ ).

\subsection{Spearman Correlation of Malat1 and THRIL IncRNAs with NIHSS and sampling time after stroke}

Moreover, the Spearman correlation between the expression levels of Malat1 and THRIL with NIHSS score in the IS patients was also detected. As shown in Fig 2A, the level of Malat1 expression in all of the patients $(n=59)$ was not correlated with NIHSS scores $(r=-0.11,95 \% C L=-0.36$ to 0.14$), p=0.36$. We also found non-significant positive correlation between THRIL expression and stroke severity. $(r=0.24$, $95 \% \mathrm{CL}=-0.015$ to 0.46$), p=0.06$ (Fig. $2 \mathrm{~B}$ ). 


\subsection{Diagnostic value and prediction of functional outcome of Malat1 and THRIL expression levels in peripheral blood of IS patients}

ROC curve analyses were used to evaluate the diagnostic value of Malat1 and THRIL IncRNAs for discriminating IS patients from the controls and revealed that Malat1 could be used as a diagnostic marker with an AUC of $0.78, p<0.001$ (Fig $3, A$ ). The sensitivity and specificity were $79.6 \%$ and $71.2 \%$, respectively.

ROC curve analysis of THRIL expression between cases and controls revealed that THRIL had a potential for discriminating IS patients from controls with an AUC of $0.50, p=0.9$, and the sensitivity and specificity (47.5\% and $45.8 \%$ ) respectively (Fig 3. B).

In our study, mRS score of 3-6 in 3 months after stroke was considered as an unfavorable functional outcome. Using a ROC curve analysis, Malat1 and THRIL levels showed no significant predictive prognosis for a 3-month unfavorable outcome relative to a favorable outcome with an AUC $=0.51,(95 \%$ $\mathrm{Cl}, 0.36$ to 0.66$), p=0.8$ and $\mathrm{AUC}=0.50(95 \% \mathrm{Cl}, 0.34$ to 0.65$), p=0.9$ respectively (Fig3. $\mathrm{C}$ and $\mathrm{D}$ ). The sensitivity and specificity were $(54.2 \%$ and $54.3 \%)$ and $(51.4 \%$ and $54.1 \%)$ respectively.

\section{Discussion}

In the past decade, authors have identified the changes in the expression level of different IncRNAs after IS and also have evaluated the potential of different IncRNAs as a diagnostic and prognostic marker in IS $[2,5,47,48]$. We decided to evaluate the expression level of Malat 1 and THRIL on days 1,3 , and 5 after stroke and their correlations with clinical parameters as well as their potential as diagnostic and prognostic markers in patients with LAA and SVD stroke.

In this study, we evaluated the Malat1 and THRIL expression in IS patients with LAA and SVD subtypes, while associated risk factor in LAA and SVD has been reported diabetes and hypertension respectively [49] therefore, it may be sufficient to justify this significant difference between cases and controls. Linear regression analysis did not show a significant association between expression of Malat1 and THRIL with diabetes and hypertension in IS patients. Thus, it does not appear to function as interfering factors with the expression of Malat1 and THRIL after stroke.

Our results indicated that the relative level of Malat1 significantly was elevated in peripheral blood of IS patients (LAA, SVD) compared to controls. The protective, anti-apoptotic, and anti-inflammatory roles of Malat1 in brain microvasculature and animal model were reported by Zhang et al. in 2017 [50]. In another study has been demonstrated that Malat1 was significantly upregulated in vitro and in vivo models of 
IR/I and the inhibition of Malat1 could alleviate astrocyte cell injury, reduce brain injury and neurological behavior [13]. On the other hand, downregulation and the protective role of Malat1 in IS patients has been shown by Ren et.al in 2020 [37]. According to the above-mentioned, there is some controversy about Malat1 expression and its effects after IR/I. The protective roles of IncRNA Malat1 in neurological or cerebrovascular diseases were demonstrated through activating phosphatidylinositol 3-kinase (PI3K) [51] via inhibition of proapoptotic or pro-inflammatory factors [50, 52].

Ren et al. (2020) found that Malat1 was decreased in peripheral blood of IS patients relative to controls within $24 \mathrm{~h}$ after stroke while it had a protective effect, although they found a significant elevation in its relative expression in diabetic IS compared to non-diabetic patients. We could show the high expression level of Malat1 on days 1,3, and 5 in 59 patients after stroke without significant difference between diabetic and non-diabetic patients, and this high expression level of IncRNA-Malat1 was not correlated with the NIHSS score.

According to previous evidence that demonstrated Malat1 and THRIL expressions were upregulated in atherosclerotic patients with coronary artery diseases $[18,19,21,28]$ we hypothesized that in patients with atherosclerotic IS Malat1 and THRIL expression may be upregulated although present results supported our hypothesis this is in contradiction with Ren's study, This controversy could be attributed to the types of stroke and/or time of blood sampling. We had 59 IS cases with LAA and SVD while in Ren's study the Malat1 level was assessed in 120 IS patients with all types of stroke. We found a significant positive correlation between Malat1 expression level with the timing of sampling within $24 \mathrm{~h}$ after stroke on the other hand mix model analysis has been also shown a significant decrease of Malat1 expression on day 1 relatives to the expression level on day 5 . In our study, most patients were admitted while more than $12 \mathrm{~h}$ had elapsed from their stroke symptoms and blood samplings were repeated on days 3 and 5 after stroke. The first hours after the onset of stroke have been shown as the optimal time window in previous studies $[53,54]$. Therefore, maybe in the first hours $(<12 \mathrm{~h})$ after stroke onset, the Malat1 expression was lower than the next hours. A significant negative correlation was detected between Malat1 expression and NIHSS in Ren's study but we found non -significant negative Spearman correlations $(r=-0.11, p=0.36)$ between Malat1 expression and NIHSS score, and also linear regression showed a non-significant negative relationship between Malat1 expression with NIHSS and aspect. This represents the possible protective role of Malat1 following IS. Therefore, it is possible that evaluation of the Malat1 expression in a larger sample size and in the optimal time window may be showing its significant correlation with NIHSS.

For the first time, we evaluated the THRIL expression in peripheral blood of IS patients. In our study, THRIL was significantly upregulated on the first day after IS and its expression level was higher on day 5 compared to THRIL level on days 1 and 3 and we could find only a non-significant positive correlation between THRIL expression and stroke severity.

Previous studies showed that following in vitro and in vivo models of cerebral ischemia-reperfusion injury THRIL expression was upregulated $[27,55]$. Hypoxia-induced increase of THRIL expression and the 
protective role of THRIL inhibition against hypoxia-induced injuries has been demonstrated in-vitro studies $[55,56]$. THRIL inhibition increased cell viability, migration, and invasion, and decreased cell apoptosis by up-regulating miR-99a expression in H9C2 cells [55]. THRIL could aggravate the cerebral IR/I by binding to miR-24-3p and activation of the NF-KB p65 signaling pathway [27]. Lin et al. reported the alleviation of myocardial injury after THRIL inhibition in the coronary heart disease model [57].

ROC curve analysis showed the high expression level of Malat1 within $24 \mathrm{~h}$ after stroke (more than $12 \mathrm{~h}$ ) in patients with LAA and SVD stroke had the diagnostic value with AUC of $0.78(p<0.001)$ relative to control but in Rens study the low level of Malat1 in IS patients relative to control in the first day after stroke showed diagnostic value with AUC of 0.79. In Ren's study, the expression of Malat1 in different types of stroke was not compared.

This was the first study evaluating the expression level of THRIL in IS patients. The upregulation of THRIL with an AUC of 0.5 did not show a significant diagnostic value and we also found a non-significant positive correlation between THRIL expression and stroke severity in clinical practice $(r=0.24, p=0.06)$.

To reach a reliable conclusion we need a larger sample size that simultaneously assesses the expression level of Malat1 and THRIL in the first hours after stroke in all types of stroke. The possible mechanisms of Malat1 and THRIL in the pathophysiology of stroke should be conducted in the future. Taken together, the upregulation of THRIL after stroke may serve as a biomarker for the diagnosis of IS.

\section{Declarations}

\section{Funding:}

This work was supported by Shiraz University of Medical Sciences (IR.SUMS.REC.1398. with grant agreement No. 17988).

\section{Compliance with Ethical Standards}

\section{Conflict of Interest}

The authors declare no competing interests.

\section{Ethical standard.}

This study has been approved by the local ethics committee of Shiraz University of Medical Sciences and have been performed in accordance with the ethical standards laid down in the 1964 Declaration of Helsinki and its later amendments. 


\section{Consent to participate.}

Written informed consent was obtained from all patients included in this study before blood sampling.

\section{References}

1. Hankey GJ, Spiesser J, Hakimi Z, Bego G, Carita P, Gabriel S (2007) Rate, degree, and predictors of recovery from disability following ischemic stroke. Neurology 68(19):1583-7. doi:

10.1212/01.wnl.0000260967.77422.97

2. Jickling GC, Sharp FR (2011) Blood biomarkers of ischemic stroke. Neurotherapeutics 8(3):349-360. doi: 10.1007/s13311-011-0050-4

3. Statello L, Guo CJ, Chen LL, Huarte M (2021) Gene regulation by long non-coding RNAs and its biological functions. Nat Rev Mol Cell Biol 22(2):96-118. doi: 10.1038/s41580-020-00315-9

4. Gomes CPC, Spencer H, Ford KL, Michel LYM, Baker AH, Emanueli C et al (2017) The Function and Therapeutic Potential of Long Non-coding RNAs in Cardiovascular Development and Disease. Mol Ther Nucleic Acids 8:494-507. doi: 10.1016/j.omtn.2017.07.014

5. Bao MH, Szeto V, Yang BB, Zhu SZ, Sun HS, Feng ZP (2018) Long non-coding RNAs in ischemic stroke. Cell Death Dis 9(3):281. doi: 10.1038/s41419-018-0282-x

6. Chen R, Xu X, Huang L, Zhong W, Cui L (2019) The Regulatory Role of Long Noncoding RNAs in Different Brain Cell Types Involved in Ischemic Stroke. Front Mol Neurosci 12:61. doi: 10.3389/fnmol.2019.00061

7. Djebali S, Davis CA, Merkel A, Dobin A, Lassmann T, Mortazavi A et al (2012) Landscape of transcription in human cells. Nature 489(7414):101-108. doi: 10.1038/nature11233

8. Zhao Y, Yu YQ, You S, Zhang CM, Wu L, Zhao W et al (2020) Long Non-Coding RNA MALAT1 as a Detection and Diagnostic Molecular Marker in Various Human Cancers: A Pooled Analysis Based on 3255 Subjects. Onco Targets Ther 13:5807-5817. doi: 10.2147/OTT.S250796

9. Ji P, Diederichs S, Wang W, Boing S, Metzger R, Schneider PM et al (2003) MALAT-1, a novel noncoding RNA, and thymosin beta4 predict metastasis and survival in early-stage non-small cell lung cancer. Oncogene 22(39):8031-8041. doi: 10.1038/sj.onc.1206928

10. Arun G, Diermeier S, Akerman M, Chang KC, Wilkinson JE, Hearn S et al (2016) Differentiation of mammary tumors and reduction in metastasis upon Malat1 IncRNA loss. Genes Dev 30(1):34-51. doi: $10.1101 /$ gad. 270959.115

11. Biswas S, Thomas AA, Chen S, Aref-Eshghi E, Feng B, Gonder J et al (2018) MALAT1: An Epigenetic Regulator of Inflammation in Diabetic Retinopathy. Sci Rep 8(1):6526. doi: 10.1038/s41598-01824907-w

12. Arun G, Aggarwal D, Spector DL (2020) MALAT1 Long Non-Coding RNA: Functional Implications. Noncoding RNA 6(2). doi: 10.3390/ncrna6020022 
13. Jin J, Wang $H$, Zheng $X$, Xie S, Zheng L, Zhan R (2020) Inhibition of LncRNA MALAT1 Attenuates Cerebral Ischemic Reperfusion Injury via Regulating AQP4 Expression. Eur Neurol 83(6):581-590. doi: $10.1159 / 000511238$

14. Huang K, Yu X, Yu Y, Zhang L, Cen Y, Chu J (2020) Long noncoding RNA MALAT1 promotes high glucose-induced inflammation and apoptosis of vascular endothelial cells by regulating miR-3613p/SOCS3 axis. Int J Clin Exp Pathol 13(5):1243-1252

15. Zhou Q, Run Q, Li CY, Xiong XY, Wu XL (2020) LncRNA MALAT1 Promotes STAT3-Mediated Endothelial Inflammation by Counteracting the Function of miR-590. Cytogenet Genome Res 160(10):565-578. doi: 10.1159/000509811

16. Patel NA, Moss LD, Lee JY, Tajiri N, Acosta S, Hudson C et al (2018) Long noncoding RNA MALAT1 in exosomes drives regenerative function and modulates inflammation-linked networks following traumatic brain injury. J Neuroinflammation 15(1):204. doi: 10.1186/s12974-018-1240-3

17. Chen $H$, Wang $X$, Yan X, Cheng X, He X, Zheng W (2018) RETRACTED: LncRNA MALAT1 regulates sepsis-induced cardiac inflammation and dysfunction via interaction with miR-125b and p38 MAPK/NFkappaB. Int Immunopharmacol 55:69-76. doi: 10.1016/j.intimp.2017.11.038

18. Toraih EA, El-Wazir A, Alghamdi SA, Alhazmi AS, El-Wazir M, Abdel-Daim MM et al (2019) Association of long non-coding RNA MIAT and MALAT1 expression profiles in peripheral blood of coronary artery disease patients with previous cardiac events. Genet Mol Biol 42(3):509-518. doi: 10.1590/16784685-GMB-2018-0185

19. Zhao C, Zong Z, Zhu Q, Wang Y, Li X, Zhang C et al (2021) The IncRNA MALAT1 participates in regulating coronary slow flow endothelial dysfunction through the miR-181b-5p-MEF2A-ET-1 axis. Vascul Pharmacol 138:106841. doi: 10.1016/j.vph.2021.106841

20. Huangfu N, Xu Z, Zheng W, Wang Y, Cheng J, Chen X (2018) LncRNA MALAT1 regulates oxLDLinduced CD36 expression via activating beta-catenin. Biochem Biophys Res Commun 495(3):21117. doi: $10.1016 / j . b b r c .2017 .12 .086$

21. Qiu S, Sun J (2020) IncRNA-MALAT1 expression in patients with coronary atherosclerosis and its predictive value for in-stent restenosis. Exp Ther Med 20(6):129. doi: 10.3892/etm.2020.9258

22. Xu B, Jin X, Yang T, Zhang Y, Liu S, Wu L et al (2020) Upregulated IncRNA THRIL/TNF-alpha Signals Promote Cell Growth and Predict Poor Clinical Outcomes of Osteosarcoma. Onco Targets Ther 13:119-129. doi: 10.2147/OTT.S235798

23. Li Z, Chao TC, Chang KY, Lin N, Patil VS, Shimizu C et al (2014) The long noncoding RNA THRIL regulates TNFalpha expression through its interaction with hnRNPL. Proc Natl Acad Sci U S A 111(3):1002-1007. doi: 10.1073/pnas.1313768111

24. Liu T, Liu J, Tian C, Wang $H$, Wen M, Yan M (2020) LncRNA THRIL is upregulated in sepsis and sponges miR-19a to upregulate TNF-alpha in human bronchial epithelial cells. J Inflamm (Lond) 17:31. doi: 10.1186/s12950-020-00259-z

25. Qi H, Shen J, Zhou W (2020) Up-regulation of long non-coding RNA THRIL in coronary heart disease: Prediction for disease risk, correlation with inflammation, coronary artery stenosis, and major 
adverse cardiovascular events. J Clin Lab Anal 34(5):e23196. doi: 10.1002/jcla.23196

26. Moharamoghli M, Hassan-Zadeh V, Dolatshahi E, Alizadeh Z, Farazmand A (2019) The expression of GAS5, THRIL, and RMRP IncRNAs is increased in T cells of patients with rheumatoid arthritis. Clin Rheumatol 38(11):3073-3080. doi: 10.1007/s10067-019-04694-z

27. Kuai F, Zhou L, Zhou J, Sun X, Dong W (2021) Long non-coding RNA THRIL inhibits miRNA-24-3p to upregulate neuropilin-1 to aggravate cerebral ischemia-reperfusion injury through regulating the nuclear factor kappaB p65 signaling. Aging 13(6):9071-9084. doi: 10.18632/aging.202762

28. Xiao J, Lu Y, Yang X (2020) THRIL mediates endothelial progenitor cells autophagy via AKT pathway and FUS. Mol Med 26(1):86. doi: 10.1186/s10020-020-00201-2

29. Zhu Y, Yang T, Duan J, Mu N, Zhang T (2019) MALAT1/miR-15b-5p/MAPK1 mediates endothelial progenitor cells autophagy and affects coronary atherosclerotic heart disease via mTOR signaling pathway. Aging 11(4):1089-1109. doi: 10.18632/aging.101766

30. Gao H, Wang X, Lin C, An Z, Yu J, Cao H et al (2020) Exosomal MALAT1 derived from ox-LDL-treated endothelial cells induce neutrophil extracellular traps to aggravate atherosclerosis. Biol Chem 401(3):367-376. doi: 10.1515/hsz-2019-0219

31. Cai Q, Gao ML, Huang LS, Chen HS, Pan LH (2021) MALAT1/miRNA-203/Wnt5a: A potential mechanism for regulating coronary artery disease. Int J Cardiol 329:48. doi:

10.1016/j.ijcard.2020.12.046

32. Liu JY, Yao J, Li XM, Song YC, Wang XQ, Li YJ et al (2014) Pathogenic role of IncRNA-MALAT1 in endothelial cell dysfunction in diabetes mellitus. Cell Death Dis 5:e1506. doi:

10.1038/cddis.2014.466

33. Han Y, Qiu H, Pei X, Fan Y, Tian H, Geng J (2018) Low-dose Sinapic Acid Abates the Pyroptosis of Macrophages by Downregulation of IncRNA-MALAT1 in Rats With Diabetic Atherosclerosis. $J$ Cardiovasc Pharmacol 71(2):104-112. doi: 10.1097/FJC.0000000000000550

34. Powers WJ, Rabinstein AA, Ackerson T, Adeoye OM, Bambakidis NC, Becker K et al (2019) Guidelines for the Early Management of Patients With Acute Ischemic Stroke: 2019 Update to the 2018 Guidelines for the Early Management of Acute Ischemic Stroke: A Guideline for Healthcare Professionals From the American Heart Association/American Stroke Association. Stroke 50(12):e344-e418. doi: 10.1161/STR.0000000000000211

35. Li H, Zhu X, Hu L, Li Q, Ma J, Yan J (2019) Loss of exosomal MALAT1 from ox-LDL-treated vascular endothelial cells induces maturation of dendritic cells in atherosclerosis development. Cell Cycle 18(18):2255-2267. doi: 10.1080/15384101.2019.1642068

36. Lv F, Liu L, Feng Q, Yang X (2021) Long non-coding RNA MALAT1 and its target microRNA-125b associate with disease risk, severity, and major adverse cardiovascular event of coronary heart disease. J Clin Lab Anal 35(4):e23593. doi: 10.1002/jcla.23593

37. Ren H, Wu F, Liu B, Song Z, Qu D (2020) Association of circulating long non-coding RNA MALAT1 in diagnosis, disease surveillance, and prognosis of acute ischemic stroke. Braz J Med Biol Res 53(12):e9174. doi: 10.1590/1414-431X20209174 
38. Borhani-Haghighi A, Safari R, Heydari ST, Soleimani F, Sharifian M, Yektaparast Kashkuli S et al (2013) Hospital mortality associated with stroke in southern iran. Iran J Med Sci 38(4):314-320

39. Tabrizi R, Lankarani KB, Kardeh B, Akbari H, Azarpazhooh MR, Borhani-Haghighi A (2021) A Comprehensive Systematic Review and Meta-analysis on the Risk Factors of Stroke in Iranian Population. Arch Iran Med 24(1):64-77. doi: 10.34172/aim.2021.10

40. Ebrahimi M, Kazemi-Bajestani SM, Ghayour-Mobarhan M, Ferns GA (2011) Coronary artery disease and its risk factors status in iran: a review. Iran Red Crescent Med J 13(9):610-623. doi:

10.5812/kowsar.20741804.2286

41. Adams HP Jr, Bendixen BH, Kappelle LJ, Biller J, Love BB, Gordon DL et al (1993) Classification of subtype of acute ischemic stroke. Definitions for use in a multicenter clinical trial. TOAST. Trial of Org 10172 in Acute Stroke Treatment. Stroke 24(1):35-41. doi: 10.1161/01.str.24.1.35

42. Unger T, Borghi C, Charchar F, Khan NA, Poulter NR, Prabhakaran D et al (2020) 2020 International Society of Hypertension Global Hypertension Practice Guidelines. Hypertension 75(6):1334-1357. doi: 10.1161/HYPERTENSIONAHA. 120.15026

43. American Diabetes A (2004) Diagnosis and classification of diabetes mellitus. Diabetes Care 27(Suppl 1):S5-S10. doi: 10.2337/diacare.27.2007.s5

44. Williams LS, Yilmaz EY, Lopez-Yunez AM (2000) Retrospective assessment of initial stroke severity with the NIH Stroke Scale. Stroke 31(4):858-862. doi: 10.1161/01.str.31.4.858

45. Nunn A, Bath PM, Gray LJ (2016) Analysis of the Modified Rankin Scale in Randomised Controlled Trials of Acute Ischaemic Stroke: A Systematic Review. Stroke Res Treat 2016:9482876. doi: $10.1155 / 2016 / 9482876$

46. Schmittgen TD, Livak KJ (2008) Analyzing real-time PCR data by the comparative C T method. Nat Protoc 3(6):1101

47. Zhu M, Li N, Luo P, Jing W, Wen X, Liang C et al (2018) Peripheral blood leukocyte expression of IncRNA MIAT and its diagnostic and prognostic value in ischemic stroke. J Stroke Cerebrovasc Dis 27(2):326-337

48. Rezaei M, Mokhtari MJ, Bayat M, Safari A, Dianatpuor M, Tabrizi R et al (2021) Long non-coding RNA $\mathrm{H} 19$ expression and functional polymorphism rs217727 are linked to increased ischemic stroke risk. BMC Neurol 21(1):54. doi: 10.1186/s12883-021-02081-3

49. Harris S, Sungkar S, Rasyid A, Kurniawan M, Mesiano T, Hidayat R (2018) TOAST Subtypes of Ischemic Stroke and Its Risk Factors: A Hospital-Based Study at Cipto Mangunkusumo Hospital, Indonesia. Stroke Res Treat 2018:9589831. doi: 10.1155/2018/9589831

50. Liu C, Zhang C, Yang J, Geng X, Du H, Ji X et al (2017) Screening circular RNA expression patterns following focal cerebral ischemia in mice. Oncotarget 8(49):86535

51. Xin JW, Jiang YG (2017) Long noncoding RNA MALAT1 inhibits apoptosis induced by oxygenglucose deprivation and reoxygenation in human brain microvascular endothelial cells. Exp Ther Med 13(4):1225-1234. doi: 10.3892/etm.2017.4095 
52. Masoumi F, Ghorbani S, Talebi F, Branton WG, Rajaei S, Power C et al (2019) Malat1 long noncoding RNA regulates inflammation and leukocyte differentiation in experimental autoimmune encephalomyelitis. J Neuroimmunol 328:50-59. doi: 10.1016/j.jneuroim.2018.11.013

53. Wang J, Zhao H, Fan Z, Li G, Ma Q, Tao Z et al (2017) Long Noncoding RNA H19 Promotes Neuroinflammation in Ischemic Stroke by Driving Histone Deacetylase 1-Dependent M1 Microglial Polarization. Stroke 48(8):2211-2221. doi: 10.1161/STROKEAHA.117.017387

54. Xiao Z, Qiu Y, Lin Y, Medina R, Zhuang S, Rosenblum JS et al (2019) Blocking IncRNA H19-miR-19aId2 axis attenuates hypoxia/ischemia induced neuronal injury. Aging 11(11):3585-3600. doi: 10.18632/aging.101999

55. Xia J, Jiang N, Li Y, Wei Y, Zhang X (2019) The long noncoding RNA THRIL knockdown protects hypoxia-induced injuries of H9C2 cells through regulating miR-99a. Cardiol J 26(5):564-574. doi: 10.5603/CJ.a2018.0054

56. Sheng C, Hu F, Wu L (2019) Geniposide alleviates hypoxia-induced injury by down-regulation of IncRNA THRIL in rat cardiomyocytes derived H9c2 cells. Eur J Pharmacol 854:28-38. doi:

10.1016/j.ejphar.2019.03.058

57. Lin L, Bao J (2021) Long non-coding RNA THRIL is upregulated in coronary heart disease and binds to microRNA-424 to upregulate TXNIP in mice. Microvasc Res 138:104215. doi:

10.1016/j.mvr.2021.104215

\section{Tables}

\section{Table 1}

Demographic and clinical characteristics of the patients and controls. 


\section{Characteristics}

Male, n (\%)

Female, n (\%)

Age, years

$\mathrm{BMI}\left(\mathrm{kg} / \mathrm{m}^{2}\right)$

NIHSS at admission

$\leq 6$

$\geq 7$

Aspect

$<6$

$\geq 6$

mRS at admission

0-2

3-6

mRS at 3 months

0-2

3-6

Vascular risk factors

Hypertension, n (\%)

Diabetes, n (\%)

Smoking, n (\%)

Drinking, n (\%)

Hyperlipidemia

Laboratory findings

Cases $(\mathrm{n}=59) \quad$ Controls $(\mathrm{n}=65) \quad P$

$42(71.2 \%) \quad 43(68.25 \%) \quad 0.9^{a}$

$17(28.8 \%) \quad 20(31.75 \%)$

$\begin{array}{lll}63.9 \pm 1.8 & 64.66 \pm 1.7 & 0.9^{\mathrm{b}}\end{array}$

$26.15 \pm 0.6$

$25.81 \pm 3.97$

$0.9^{b}$

$22(37.3 \%)$

$37(62.7 \%)$

$14(23.7 \%)$

$45(76.3 \%)$

12 (20.33\%)

47 (79.66\%)

24(40.67\%)

$35(59.32 \%)$ 
Triglyceride, $\mathrm{mg} / \mathrm{dL}$

Total Cholesterol, $\mathrm{mg} / \mathrm{dL}$

Low Density Lipoprotein, mg/dL

High Density Lipoprotein, mg/dL
126. $7 \pm 7.08$

$161.2 \pm 5.6$

$97.6 \pm 4.5$

$33.7 \pm 0.9$
$132.81 \pm 9.8$

$0.6^{\mathrm{b}}$

$162.19 \pm 4.1$

$0.9^{b}$

$90.60 \pm 3.7$

$0.2^{b}$

$44.21 \pm 1.5$

$0.003^{b}$

Types of stroke

Large artery atherosclerosis n (\%)

$29(49.2 \%)$

Small-vessel disease $\mathrm{n}(\%)$

$30(50.8 \%)$

Data were shown as mean \pm SEM or as $\mathrm{n}(\%) .{ }^{\mathrm{a}}$ Chi-square Test; ${ }^{\mathrm{b}}$ Independent two-sample T-test. IS, ischemic stroke; $\mathrm{BMI}$, body mass index;

NIHSS, National Institutes of Health Stroke Scale; mRS, modified Rankin Scale,

\section{Table 2}

Time interaction for Malat1 and THRIL expression was estimated by mixed model analysis after adjusting the group.

\begin{tabular}{lllllllll} 
Parameter & Estimate & $p$ & $95 \% \mathrm{Cl}$ & \multicolumn{3}{c}{ Estimate } & $p$ & $95 \% \mathrm{Cl}$ \\
& THRIL & & Lower & Upper & Malat1 & & Lower & Upper \\
\hline Intercept & 9.64 & .000 & 7.08 & 12.20 & 6.80 & .000 & 4.91 & 8.69 \\
\hline (Day 1) & -6.47 & .000 & -9.36 & -3.59 & -2.69 & .011 & -4.77 & -.62 \\
\hline (Day 3) & -5.67 & .000 & -8.68 & -2.66 & -.08 & .934 & -2.24 & 2.06 \\
\hline (Day 5) Ref & $0^{\text {b }}$ & & & & $0^{\text {b }}$ &. &. &. \\
\hline [group_2=0] & -7.04 & .000 & -10.56 & -3.52 & -5.75 & .000 & -8.35 & -3.15 \\
\hline [group_2=1] & $0^{\text {b }}$ &. &. &. & $0^{\text {b }}$ &. &. &.
\end{tabular}

After adjusting the group, the significant time interaction was detected for Malat1 and THRIL expression. The level of THRIL

on days 1 and 3 significantly was lower than the expression on day 5 respectively and the Malat 1 level on day 1 significantly was lower than the expression on day 5 


\section{Table 3}

Linear regression analysis for the association between clinical parameters with Malat1 and THRIL IncRNA levels in ischemic stroke patients.

\begin{tabular}{|c|c|c|c|c|c|c|c|c|}
\hline \multirow[t]{2}{*}{ Variables } & Beta & P & Lower & Upper & \multicolumn{4}{|l|}{ Beta } \\
\hline & \multicolumn{4}{|c|}{ Malat1 } & THRIL & P & Lower & Upper \\
\hline \multicolumn{2}{|l|}{ Constant } & .197 & -4.953 & 23.332 & & .878 & -16.469 & 19.201 \\
\hline Age & -.102 & .572 & -.162 & .091 & .122 & .511 & -.107 & .212 \\
\hline Sex & .150 & .341 & -1.795 & 5.080 & .056 & .728 & -3.581 & 5.089 \\
\hline mRS & .224 & .201 & -.372 & 1.724 & .024 & .893 & -1.233 & 1.411 \\
\hline NIHSS & -.262 & .143 & -.458 & .068 & .158 & .390 & -.189 & .475 \\
\hline BMI & .078 & .605 & -.212 & .361 & .048 & .759 & -.306 & .417 \\
\hline DM & .137 & .452 & -2.342 & 5.176 & .114 & .542 & -3.293 & 6.187 \\
\hline HTN & .161 & .350 & -1.812 & 5.017 & .114 & .522 & -2.924 & 5.688 \\
\hline HLP & .040 & .819 & -3.289 & 4.140 & .067 & .712 & -3.818 & 5.550 \\
\hline Smoker & .055 & .714 & -3.267 & 4.732 & -.045 & .772 & -5.775 & 4.313 \\
\hline SVD & -.223 & .195 & -5.599 & 1.176 & -.114 & .520 & -5.648 & 2.896 \\
\hline IHD & .035 & .831 & -3.135 & 3.882 & .008 & .963 & -4.323 & 4.526 \\
\hline \multicolumn{9}{|c|}{$\begin{array}{l}\text { NIHSS, National Institutes of Health Stroke Scale; mRS, modified Rankin Scale, BMI, body mass index; } \\
\text { DM, diabetes mellitus; }\end{array}$} \\
\hline \multicolumn{9}{|c|}{ IHD, Ischemic heart disease, HTN, Hypertension: HLP, Hyperlipidemia; SVD, Small-vessel disease } \\
\hline
\end{tabular}

\section{Figures}



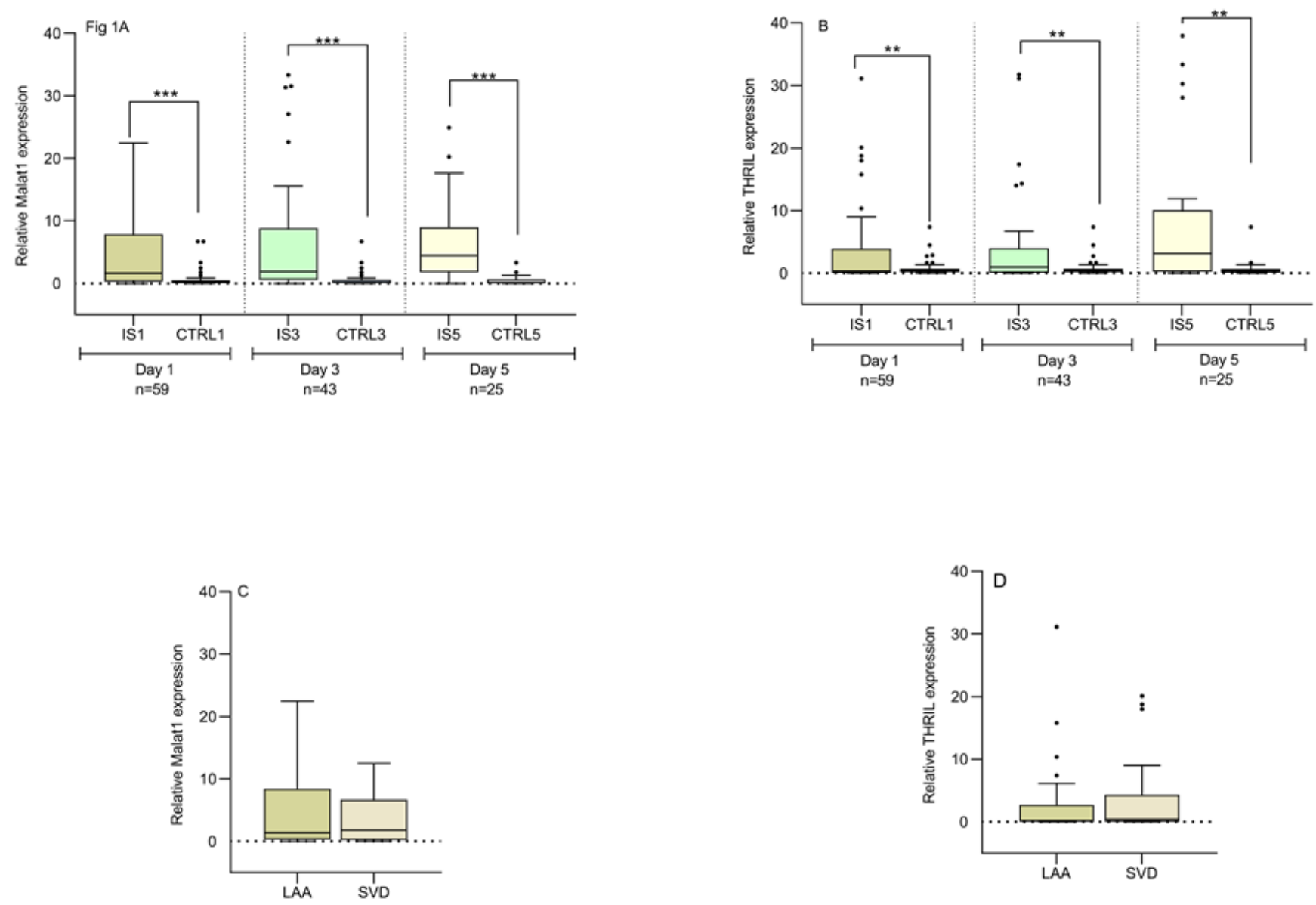

\section{Figure 1}

The expression levels of Malat1 and THRIL in patients, controls on days 1,3, and 5 after stroke. (A and B) Independent Student's t-test revealed that Malat1 and THRIL expression were significantly higher in IS patients relative to controls on days 1,3, and 5 after stroke. (C and D) The comparison of blood levels of Malat1 and THRIL in LAA and SVD. Results were expressed as mean \pm SEM. . ${ }^{\star \star} p<0.01$. ${ }^{\star \star *} p<0.001$. Abbreviations: IS, ischemic stroke; CTRL,control; LAA, large-artery atherosclerosis; SVD, small-vessel disease. 

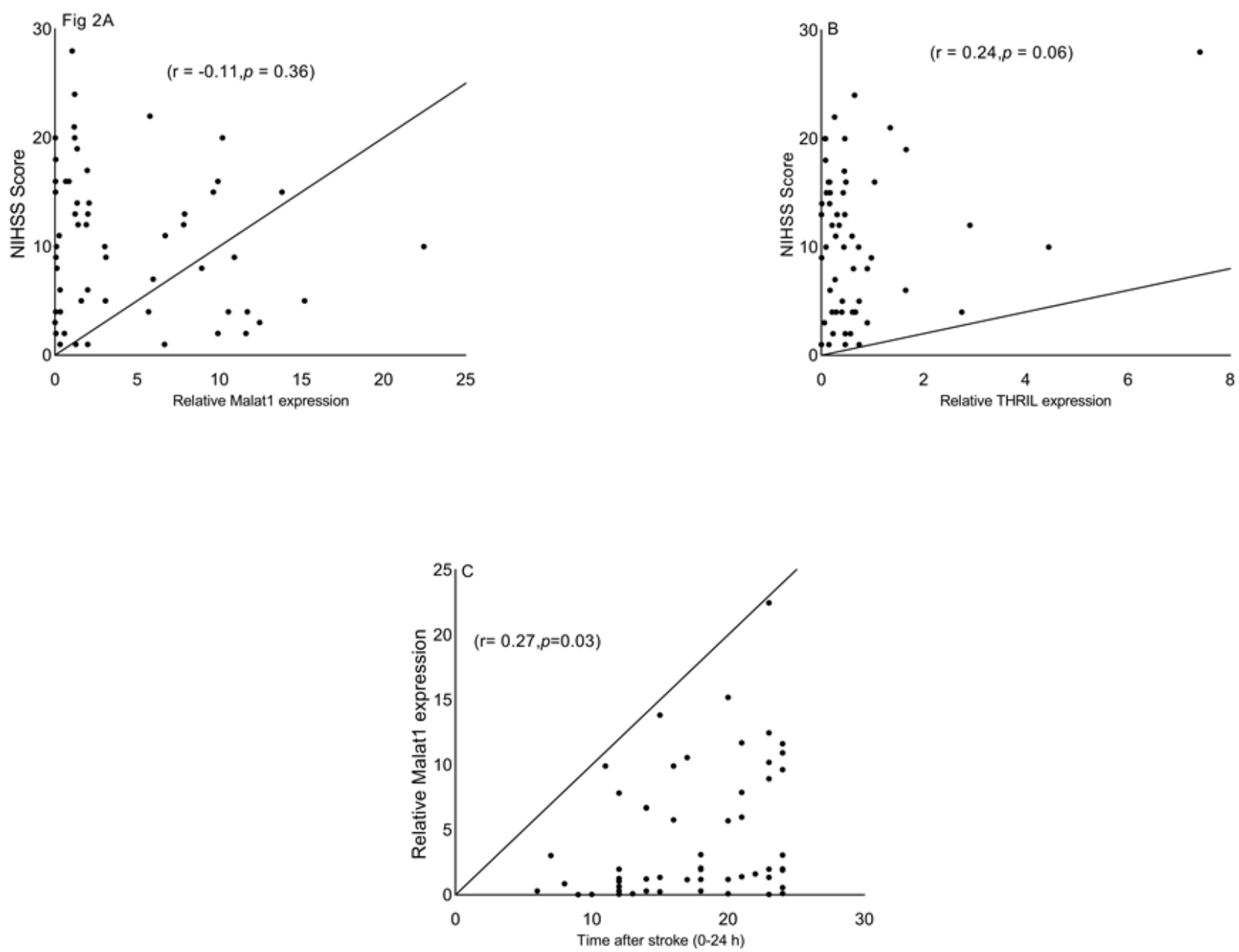

\section{Figure 2}

The Spearman Correlation between expression levels of Malat1 and THRIL with NIHSS and the time after stroke in IS patients. (A) The Spearman Correlation between expression of Malat1 and NIHSS in patients (B) between THRIL expression and NIHSS (C) between Malat1 expression and time after stroke. Abbreviations: NIHSS, National Institutes of Health Stroke Scale. 

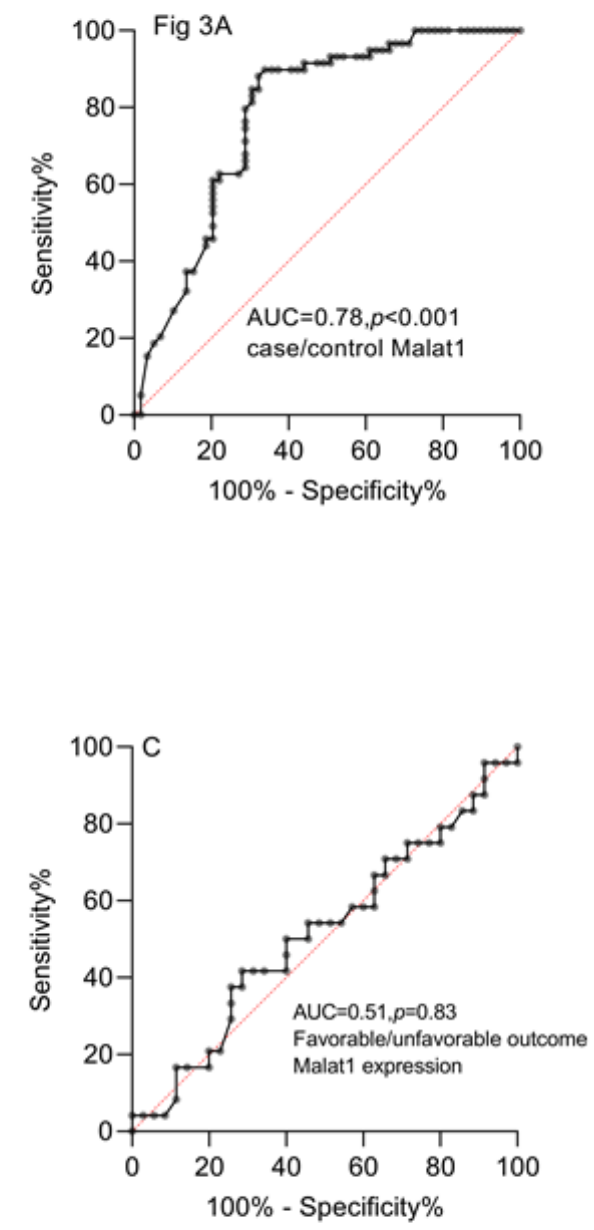
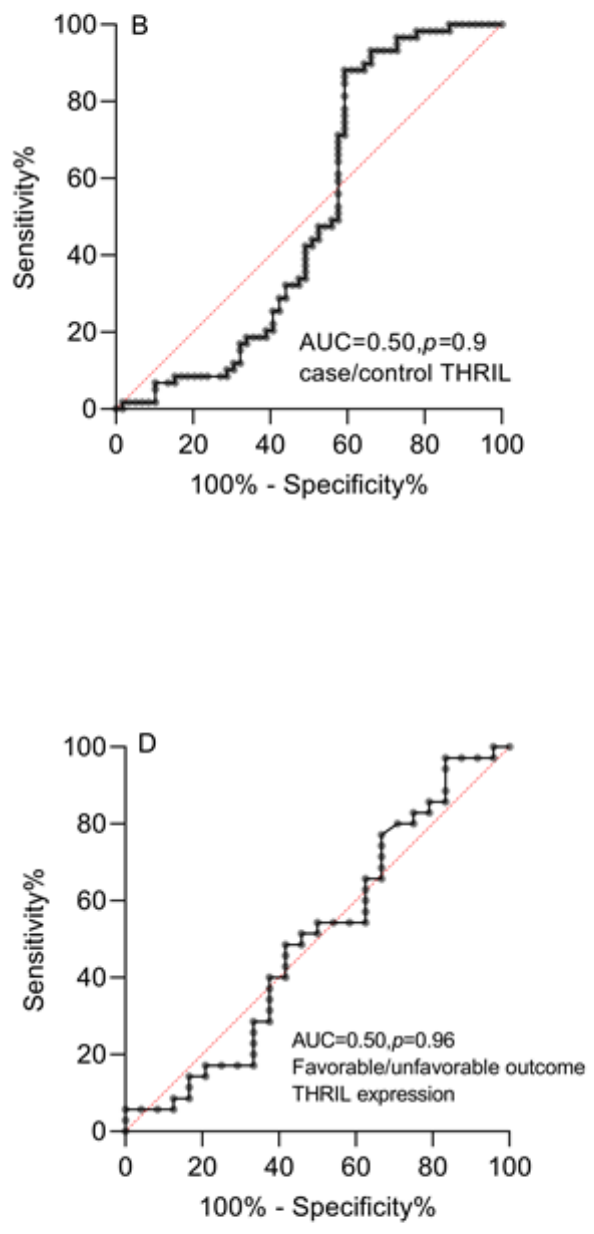

\section{Figure 3}

ROC curve analyses were used to evaluate the diagnostic and prognostic values of Malat1 and THRIL IncRNAs. (A and B) ROC curve analyses of Malat1 and THRIL for discriminating IS patients from the controls. (C and D) ROC curve analyses of Malat1 and THRIL for predictive of functional outcome. Abbreviations: ROC, receiver operating characteristic; AUC, area under the curve 